\title{
Versatile electroanalysis of cellular receptor: The case of Toll-like immune receptors evaluated on transfected human cell
}

\author{
Flávia Costa Mendonça-Natividade ${ }^{a}$, Fernanda C. Carvalho ${ }^{\mathrm{b}}$, \\ Maria-Cristina Roque-Barreira ${ }^{\mathrm{a}}$, Paulo R. Bueno ${ }^{\mathrm{b}, *}$ \\ a Cell and Molecular Biology and Pathogenic Bioagents, Ribeirão Preto Medical School, Universidade de São Paulo (University of Sao Paulo- USP), CEP \\ 14049-900, Ribeirão Preto, São Paulo, Brazil \\ ${ }^{\mathrm{b}}$ Institute of Chemistry, Physical Chemistry Department, Nanobionics Research Group, Universidade Estadual Paulista (Sao Paulo StateUniversity - \\ UNESP), CEP 355, 14800-900, Araraquara, São Paulo, Brazil ${ }^{1}$
}

\section{A R T I C L E I N F O}

\section{Article history:}

Received 27 April 2016

Received in revised form 17 October 2016

Accepted 19 October 2016

Available online 19 October 2016

\section{Keywords:}

Electrochemical impedance spectroscopy (EIS)

Biological assay

Toll-like receptor

Cell biology

\begin{abstract}
A B S T R A C T
In the present work we compare the results obtained by two approaches to detect receptor expression on cells surface: (i) the confocal laser scanning microscopy, which is an established methodology to study cell biology and associated events, wherein the cells or molecules labeling is needed, and (ii) an alternative label-free electroanalytical technique, essentially quantitative, based on Electrochemical Impedance Spectroscopy (EIS). Concerning specificity, both assays are equivalent, since they depend on the antibody efficiency to recognize and discriminate the searched structure in the cell context. Regarding sensitivity, we showed that, in the case of the detection of Toll-like receptor on transfected human cells, this label-free EIS approach (with measurements performed on $2 \%$ of paraformaldehyde, an environment that retained morphology and antigenic sites of cells), was very sensitive for the receptor detection, in a demonstration that the proposed electrical impedance analytical assays can help optimizing the ability to evaluate cellular biological events. It provides to biologists and medical community an alternative and either quantitive analytical tool that can be applied to the biological model of interest and used to design and perform a rapid multiplexable and automatable assaying methodology.
\end{abstract}

(C) 2016 Elsevier B.V. All rights reserved.

\section{Introduction}

Invasions by microorganisms are initially countered, in vertebrates, by innate defense mechanisms that pre-exist in all individuals and begin to act within minutes of the encounter of the host with the infectious agent [1]. Then, the innate immune system is called the front line of host defense. Receptors that activate the innate immune response recognize pathogens directly and trigger cellular response. They occur in macrophages, neutrophils, and dendritic cells [2]. A conserved evolutionarily recognition and signaling system was originally discovered on account of this role in embryonic development in the fruitfly Drosophila melanogaster.

Abbreviations: TLR, toll-like receptor; PAMPs, pathogen-associated molecular patterns; PRRs, pattern recognition receptors; TIR, toll/IL-1R domain; ELISA, enzyme linked immuno sorbent assay.

* Corresponding author.

E-mail addresses: flavia.bioquimica@usp.br (F.C. Mendonça-Natividade), nandaferdecarvalho@gmail.com (F.C. Carvalho), mcrbarre@fmrp.usp.br

(M.-C. Roque-Barreira), prbueno@iq.unesp.br (P.R. Bueno).

1 (www.nanobionics.pro.br)
It is recognized to have a critical role in defense against infection in plants, adult insects, and vertebrates, including mammals. The receptor mediating these function in Drosophila is known as Toll. The homologous proteins in mammals have been named Tolllike receptors (TLRs) [1]. The human genome encodes ten TLRs that recognize and respond to conserved microbial stimuli, known as pathogen-associated molecular patterns (PAMPs), and, therefore, described as pattern recognition receptors (PRRs) [3]. Instead, Drosophila protein Toll is activated by endogenous ligand [4]. The TLRs and Toll are type I transmembrane receptors with extracellular ligand-binding domains, a single membrane-spanning segment, and a cytosolic Toll/IL-1R (TIR) domain. TLRs can be located at the cell surface and are activated by lipid and protein ligands, and also signaling in response to non-self nucleic acids from endosomal compartments [5]. The analytical detection and quantification of biological cell events in complex biological samples lie central to early clinical diagnosis, drug design, and effective therapeutic application. Imaging modalities of analytical tools are optimal for understanding the physical features of structures involved in biological events in cells or tissues. One of the most important imaging analytical tools is based on light microscopy, more par- 
ticularly on confocal laser scanning microscopy. Confocal imaging is performed in a class of scanning microscopes in which both the illumination and detection systems are focused on the biological sample. The image is acquired from a thin section of the sample, and by scanning many thin sections through the sample; a refined three-dimensional image can be built. Confocal microscopy has greatly accelerated research involving imaging and morphometry [6]. However, data acquisition and images analysis constitute a laborious multistep process. Failure at any step, such as preparation of samples, negatively affects all subsequent steps. Fixation is a critical step, which requires good morphological preservation and, at the same time, antigenicity preservation [7]. An inherent problem with confocal microscopy is fading [8] derived from the incompatibility of some mounting media and certain types of fluorophores. Besides, the labeling process of the biological event under study must occur in a way to keep signal-to-noise ratio accurately or sensitive enough to obtain qualitative resolution of the cell biological event. Therefore, though sensitive, imaging approaches are laborious, time-consuming, and can lead to high background signals. In order to reach higher levels of sensitivity and to move to a detection format which is both potentially low-cost and portable besides of being time (speed) effective label-free electric analytical detection methods, such as those based on capacitive and impedimetric concepts [9] are exceedingly powerful and largely used in immunosensor platform [9-12]. Assays are generated by controllably immobilizing receptive biomolecules (typically antibodies, nucleic acids or peptides) on electrodes and converting the target binding event into a measurable electrical signal $[11,13,14]$. One of the most sensitive and powerful means of doing this is by electrical impedance [15]; that is through the application of a sinusoidal voltage to the supporting electrode and the measurement of resulting sinusoidal current. The limit of detection of this technique is up to femto molar. The ratio of the current/voltage gives the impedance, and this technique when evaluated, at different frequencies, when measured in an electrolyte biological media, for instance, is known as electrochemical impedance spectroscopy. The general anatomy of these electroanalytical devices is based on an electrode equipped with a recognition molecule specific for the biological event of interest. If the target analyte is captured by an electrode-immobilised probe (such as an antibody, DNA or aptamer), the impedance of the electrode-solution interface will change detectably. Binding of the bioreceptor by the molecular recognition site, for instance, can result in a local change of potential or current that can be detected by simple and very sensitive measurements [9]. Disease markers or molecules of general interest $[16,17]$ may be present only at sub-picomolar levels in biological fluid and, in some cases; only a few molecules of a pathological protein may trigger disease development. There exists, then, a need for ultrasensitive detection beyond the capabilities of current Enzyme Linked Immuno Sorbent Assay (ELISA) or confocal methodologies which are not able to achieve these levels of sensitivity. Thus we report herein the use of an electroanalytical electrochemical impedance spectroscopy (EIS) assay protocol [18] to detect receptors in transfected human cells as a quick and cheaper alternative electroanalytical methodology to replace those based on confocal imaging of the same biological event. As a proof-of-concept, we have comparatively evaluated the Tolllike receptors expression in transfected Human Embryonic Kidney (HEK293) cells by confocal imaging and by the proposed electroanalytical tool based on EIS methods (both analyses were done by using human cells). We observed a clear agreement between data obtained from both imaging and electroanalytical methodologies. The significant advantage displayed by the electroanalysis of the cellular receptor was its remarkable sensitivity, quantifiable and speed capability to detect transmembrane proteins expressed on the plasma membrane human cell.

\section{Materials and methods}

\subsection{Reagents}

The 11-mercaptoundecanoic acid, 6-mercaptohexanol, EDC, $\mathrm{NHS}, \mathrm{K}_{3}\left[\mathrm{Fe}(\mathrm{CN})_{6}\right]$, and $\mathrm{K}_{4}\left[\mathrm{Fe}(\mathrm{CN})_{6}\right] \cdot 3 \mathrm{H}_{2} \mathrm{O}$ were purchased from Sigma-Aldrich (St. Louis, MO, USA). The mouse anti-TLR2 human antibody was purchased from Abcam (Abcam, Cambridge, UK) and the secondary antibody donkey IgG anti-mouse Alexa 488conjugated was purchased from Thermo Scientific (Boston, MA, USA). Cell culture reagents were purchased from Gibco (Part of Invitrogen; Carlsbad, CA, USA) and transfection reagent Lipofectamine 2000 was purchased from Invitrogen (Carlsbad, CA, USA). Immunofluorescent slides were covered with Fluoromount from Sigma-Aldrich.All the solutions used in the analytical procedures were prepared with Milli-Q-purified water from Millipore (Billerica, MA, USA) with conductivity of $18.2 \mathrm{M} \Omega \mathrm{cm}$ at $25^{\circ} \mathrm{C}$. The antibody and cell solutions were prepared and used in $10 \mathrm{mM}$ phosphate-buffered saline (PBS; pH 7.4).

\subsection{Cell transfection}

Human embryonic kidney (HEK293) cells were cultured inDMEM Dulbecco's modified Eagle's medium, supplemented with $10 \%$ fetal bovine serum, at $37^{\circ} \mathrm{C}$ under a $5 \% \mathrm{CO}_{2}$ atmosphere. Cells were seeded in 24 -well plates $\left(1 \times 10^{5}\right.$ cells/well) the day before transfection. After overnight incubation, cells (approximately 80\% confluent) were transiently transfected with $180 \mathrm{ng}$ of TLR2 vector or empty vector using Lipofectamine 2000 as previously described [16]. The empty vector corresponds to the original pcDNA3.1 vector backbone without TLR2 gene cloning. At $24 \mathrm{~h}$ post transfection the one amount of cells was collected in the presence of cold phosphate buffer saline pH7,4 (PBS), for EIS assay. The cells were washed three times in the presence of PBS and fixed with $2 \%$ paraformaldehyde at room temperature for $20 \mathrm{~min}$, followed $0.1 \mathrm{M}$ glycine wash. Cells were counted in a Neubauer chamber (Weber Scientific International, Hamilton, NJ,USA) to adjust volumes at required concentration.

\subsection{Surface engineering and anti-TLR2 sensors functionalization}

For EIS analysis, the $2 \mathrm{~mm}$ gold electrode (Metrohm, Herisau, Switzerland) was cleaned and the surface area was determined as described in previous works [10]. The strategy adopted for the analysis of the EIS approach was based on the construction of molecular scale interface, i.e. a Self-assembled Monolayer (SAM) that was made with a $1: 10$ mixture of $1 \mathrm{mmol} \mathrm{L}^{-1} 11$ mercaptoundecanoic acid (Sigma), as an anchor, and $1 \mathrm{mmol} \mathrm{L}^{-1}$ 6 -mercaptohexanol, used as a spacer, in $200 \mu \mathrm{L}$ ethanol, during $16 \mathrm{~h}$. The electrode was washed first with pure ethanol and phosphate-buffered saline $0.1 \mathrm{~mol} \mathrm{~L}^{-1}$; pH 7.4 (PBS) and dried under nitrogen. Next, was performed the standard EDC/NHS bioconjugation chemistry; a solution of $0.4 \mathrm{~mol} \mathrm{~L}^{-1}$ EDC $(\mathrm{N}-(3-$ Dimethylaminopropyl)- $N$-ethyl carbodiimide hydrochloride) and $0.1 \mathrm{~mol} \mathrm{~L}^{-1} \mathrm{NHS}$ (N-Hydroxysuccinimide) was prepared and added to the electrode activating the terminal carboxyl groups on the thiol for $30 \mathrm{~min}$. The electrode was rinsed with PBS and the antibody (anti-TLR2 $1 \mu \mathrm{g} \mathrm{mL}^{-1}$ solution in PBS; $50 \mu \mathrm{L} /$ electrode) was immobilised on the functionalized surface, achieved after $1 \mathrm{~h}$ incubation. The remaining NHS esters were deactivated by the addition of a $1 \mathrm{moL}^{-1}$ ethanolamine solution for $5 \mathrm{~min}$, and the surface was thoroughly rinsed with deionized water. A $0.1 \%$ BSA solution was added for $30 \mathrm{~min}$ to block unspecific sites. After these procedures, the sensors functionalized were incubated with $10^{4}$ HEK293 cells per electrode. 


\subsection{Electrochemical impedance spectroscopy analysis}

EIS measurements were carried out on an AUTOLAB potentiostat (model PGSTAT30) that was controlled by the NOVA program, which was used for all electrochemical measurements. A threeelectrode setup was used for all procedures, consisting of a $2.0 \mathrm{~mm}$ diameter gold working electrode (Metrohm), a platinum mesh counter electrode, and an $\mathrm{Ag} \mid \mathrm{AgCl}, 3 \mathrm{~mol} \mathrm{~L}^{-1} \mathrm{KCl}$ reference electrode. EIS was used for characterization of the all-building receptive surface steps and binding analysis. EIS measurements were recorded in the presence of $1 \mathrm{mmol} \mathrm{L}^{-1}\left[\mathrm{Fe}(\mathrm{CN})_{6}\right]^{3-/ 4-}$ as the redox probe in PBS either used as a supporting electrolyte. The EIS were conducted in a frequency range of $0.01 \mathrm{~Hz}$ up to $1 \mathrm{MHz}$ in a steady-state potential of $\sim 0.22 \mathrm{~V}$ at the amplitude of $10 \mathrm{mV}$ (peak to peak) and were subsequently verified for compliance with linear systems theory by Kramers-Kronig by employing the FRA AUTOLAB software. The $\sim 0.22 \mathrm{~V}$ steady-state potential corresponds to the formal (or the half-wave) potential of the redox $\left[\mathrm{Fe}(\mathrm{CN})_{6}\right]^{3-/ 4-}$ probe applied. After electrochemical characterization of the functionalized electrode (to assure that the SAM was formed), the cells $\left(1 \times 10^{4}\right.$ cells in $50 \mu \mathrm{L}$ of PBS) were quiescently lying on the interface for $30 \mathrm{~min}$, followed by EIS analysis at the same conditions used for the SAM functionalization process. All measurements were performed in triplicate. The EIS analysis consist in the interpretation of the obtained a complex impedance function, $Z^{*}(\omega)=Z^{\prime}+j Z^{\prime \prime}$, which as a complex number (for each angular frequency, $\omega=2 \pi f$, where $f$ is the linear frequency of the spectra) contain a real ( $\left.Z^{\prime}\right)$ and an imaginary ( $Z$ ") components and wherein $j=\sqrt{-1}$ is the complex unitary number. One possible and typical analysis is the by using the Nyquist diagram representation of the EIS spectra. The Nyquist diagram consists in plotting the $-Z$ " versus $Z^{\prime}$ (proportionally, that is in a quadratic representation) from where it is possible to extract the main analytical parameter of the analysis, i.e. $R_{c t}$ the charge transfer resistance.

The $R_{c t}$ is measured graphically (thus approximately) in the Nyquist diagram by obtaining the diameter value (in Ohms) of the semi-circle of the real part of component of the complex impedance. Statistical analysis of the differences between the mean values of the experiments was performed by an appropriate homemade computational routine. The experiments are representative of three independents assays. Though undoubtedly powerful, the most rigorous approach of using $R_{c t}$ is limited by both the assumption of suitable equivalent circuit (and "fudge factors" that are often implemented in attaining a "good fit") and the need to acquire data across a full range of frequencies $[17,18]$. Nevertheless, either the determination of $R_{c t}$ can be approximately determined through the graphical analysis without misplaced of the statistics or yet fully optimized by using an automatized immittance function approach $[14,15,19]$.

\subsection{Confocal microscopy}

The HEK293 cells were cultured and transiently transfected as described above. At $16 \mathrm{~h}$ post-transfection, the cells were plating on glass slides $\left(6 \times 10^{4}\right.$ cells/glass slides $)$ and cultured for $24 \mathrm{~h}$, washed three times in the presence of PBS and fixed with $2 \%$ paraformaldehyde at room temperature for $20 \mathrm{~min}$, followed $0.1 \mathrm{M}$ glycine wash and, block with $1 \%$ BSA in PBS for $1 \mathrm{~h}$. Next, the glass slides were incubated at room temperature for $1 \mathrm{~h}$ with primary mouse antibody anti human-TLR2 $\left(5 \mu \mathrm{gmL}^{-1}\right)$ in PBS with $1 \%$ BSA, followed for $1 \mathrm{~h}$ of incubation with secondary donkey IgG antibody antimouse Alexa 488-conjugated $\left(2 \mu \mathrm{gmL}^{-1}\right)$ at room temperature for 30 min in PBS with $1 \%$ BSA. Among each step the glass slides were washed with PBS. Immunofluorescent slides were covered with Fluoromount. The data acquisition was performed with a Leica TCS SP5 confocal microscope (Leica Microsystems Inc.) with $488 \mathrm{~nm}$ excitation (Ar laser), and all images were acquired with a $63 \times$ glycerol immersion objective lens. The image analysis was performed with LAS AF Lite, version 2.6 (Leica Microsystems Inc., Wetzlar, Germany).

\section{Results and discussion}

The HEK293 cells are a particular cell line originally derived from human embryonic kidney cells grown in tissue culture. They were generated in 1973 after permanent transformation by exposing cells to sheared fragments of adenovirus type 5 DNA [20] and, currently this cell line has been extensively used as an expression tool for recombinant protein. Its biochemical machinery favors the expression of human proteins, including those of cells surface, secreted, large and multi-domain, which require a complex folding and post-translational modifications [21]. The main attributes that have made the HEK293 cell a popular choice among the researchers included: its quick and easy maintenance, high efficiency for transfection and protein production, faithful translation and processing proteins [20]. The growing information available from the human genome has placed emphasis upon heterologous cell expression systems as a target for structure-function studies, evaluation of novel drug targets and disease markers [22]. Herein, we performed the transfection of HEK293 cells with the TLR2 gene to evaluate their expression in the plasma membrane surface. The TLR2 distribution was preliminary revealed by the label-based confocal method. The transfected cells reaction with monoclonal unconjugated primary antibody anti-TLR2 was stained by using the Alexa-488 secondary antibody for viewing by confocal microscopy as shown in Fig. 1. The HEK293 cells transfected with the empty vector provided a faintly fluorescence [Fig. 1(a)], probably due to an insufficient effect of the used blocking agents, which did not totally avoid a residual non-specific binding of the primary or secondary antibody to the specimen. This fact is easily identified and does not lead to false negative results. Transfected cells with TLR2 gene clearly showed the expression of TLR2, particularly at the cell surface [Fig. 1(c)]. However, the confocal microscopy alone could not make surely evident the receptor detection at the surface of the plasma membrane, on which a large number of biological processes occur. These processes are difficult to image with conventional confocal microscopy techniques because details bordering the cell surface are hidden by fluorescence that derives from the bulk of the cell [23]. To avoid this kind of artifact, a more sophisticated approach is necessary. It concerns the total internal reflection fluorescence (TIRF) microscopy also known as an evanescent wave or evanescent field microscopy - that provides means of exciting selectively fluorophores near the cell surface while minimizing fluorescence from intracellular regions [23], which nonetheless is time-consuming.

Electroanalysis can be a low cost and an alternative approach to that of optical analyse in medical applications. In this way, several emerging ultrasensitive technologies exploit new labels such as metal/semiconducting nanoparticles and nanotubes or a combination of traditional tags (e.g. fluorescent dyes, electrochemical probes or enzyme labels) with new nanotechnological tools are being tested [17]. Using different nanoparticle/nanotube or DNA - barcode-based amplification strategies attomolar protein detection limits have been reported [17]. The compounding problem in many cases is the need (ideally) to perform such sensitive specific assays in the presence of the huge excesses (up to $10^{7}$-fold) of non-specific protein present in complex biological fluid. EIS derived electroanalytical assays $[15,18]$ are inherently spectroscopic (frequency resolved) and potentially exceedingly sensitive indicators of interfacial change (such as target binding with an appropriate receptor). As briefly discussed in the experimental section, in a more advanced way derived approaches $[18,19]$ allow opti- 

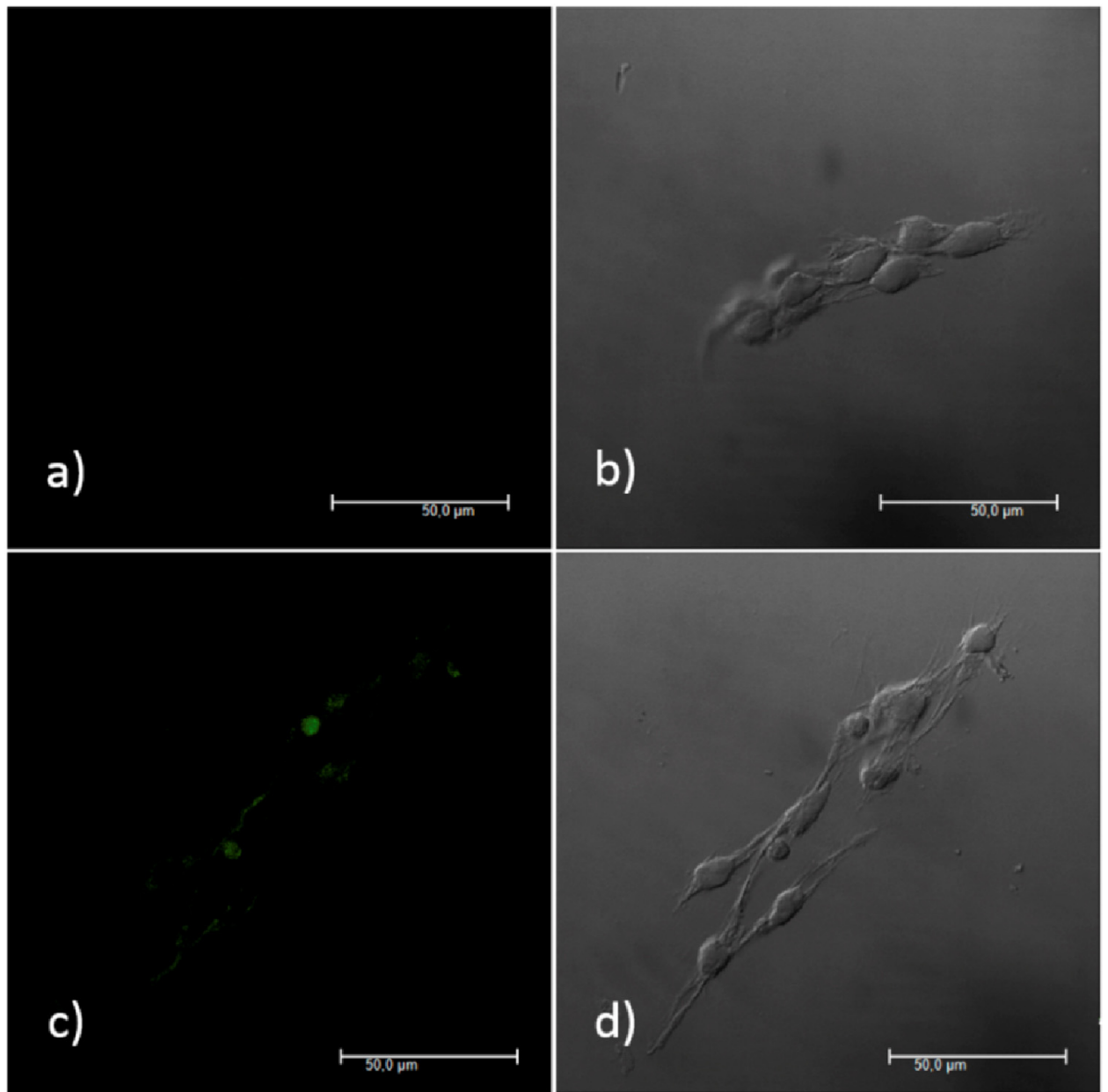

b)

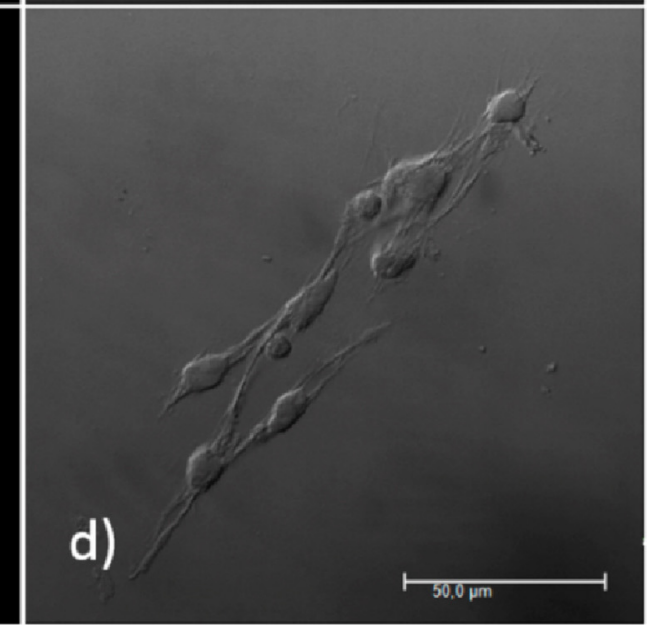

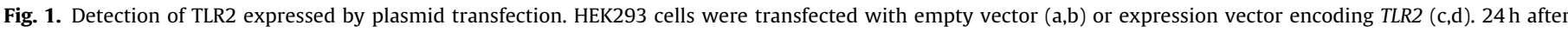

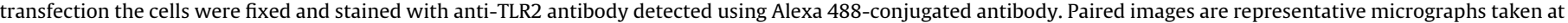
$63 \times$ objective lens acquired on Leica TCS SP5 confocal microscope.
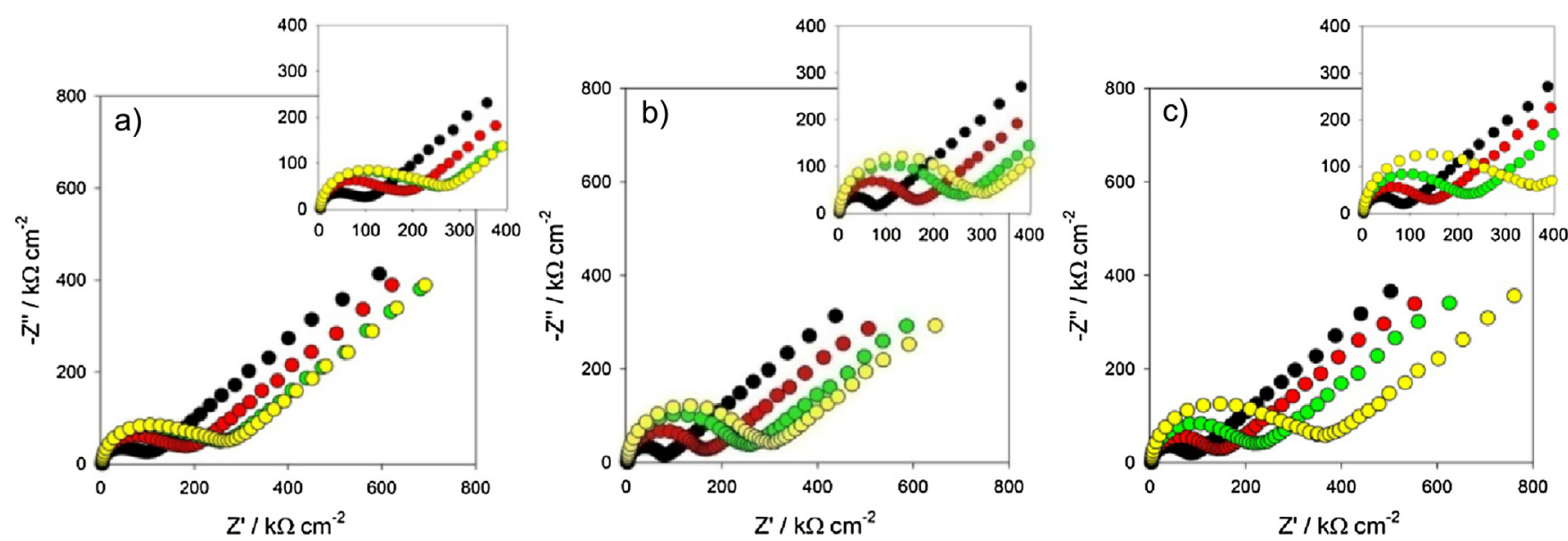

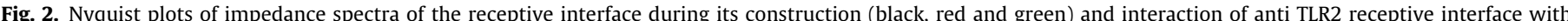

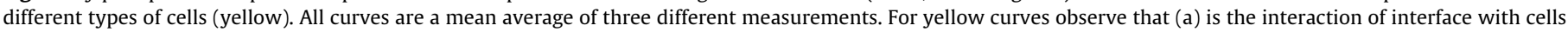

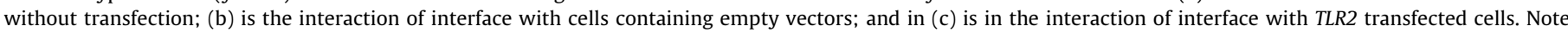

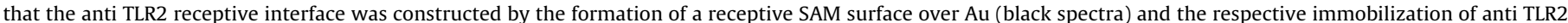

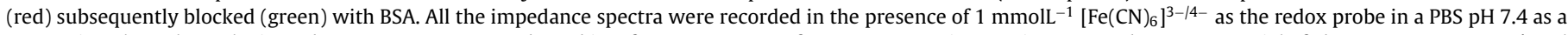

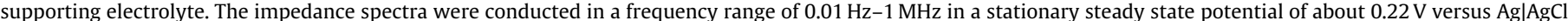

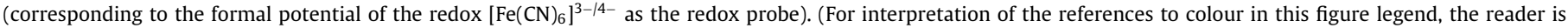
referred to the web version of this article.) 

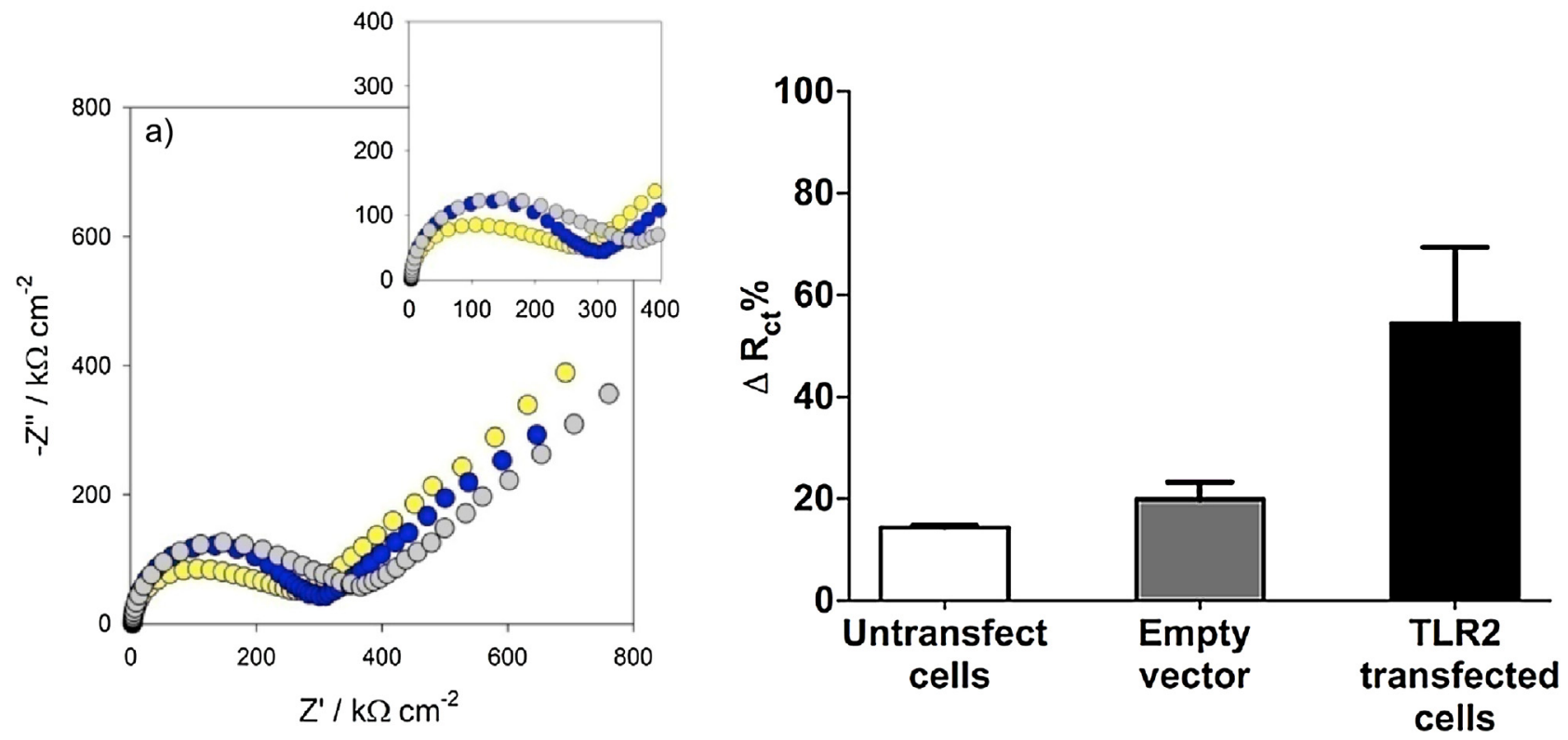

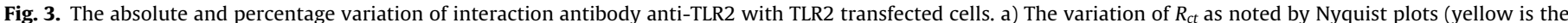

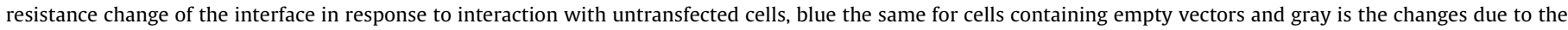

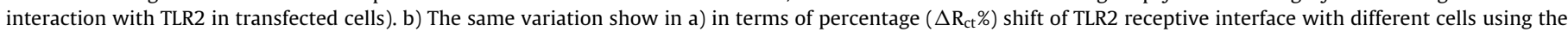

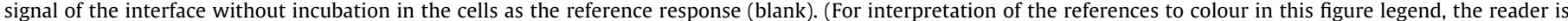
referred to the web version of this article.)

mized analytical potency and to enable the user to select and apply the most frequency-optimized reporter of interfacial change and to, thereafter, run rapid (optimized) analyses at single frequencies. EIS in its classical approach possess an innate ability to sensitively monitor conductivity/resistivity (through the use of graphically adquired $R_{c t}$ parameter - the graphical analysis avoid tedious fitting procedure with comparable statistics of the analytical curve) and is either powerful sensory tools when these surfaces are suitably receptively modified. Accordingly, the construction of the EIS-TLR2 electroanalytical sensoring surface was performed as introduced in the experimental section by monitoring how interface changes through the measurement of the charge transfer resistance $\left(R_{c t}\right)$ of the interface in Nyquist plot diagrams. The semicircles (see Fig. 2) reflect charge transfer restrictions that are sterically or electrostatically imposed as the films are being constructed or changes specifically according to the recognition or the binding advent associated with an analyte of interest, herein, for instance, the TLR on the cells' surface. Ultimately, the $R_{c t}$ is though used as a transduction signal for events of interest. Firstly, let us observe the construction of the receptive surface. This is reflected on the sharp increases of $R_{c t}$ as the receptive surface layer is gradually fabricated. As illustrated in Fig. 2(a), by adding/coupling anti-TLR (red) into the SAM monolayer (black), there is an increase of $R_{c t}$ (as said previously this can be evaluated by noting the increase of the semi-cycle in these EIS Nyquist diagrams from about $150 \mathrm{k} \Omega \mathrm{cm}^{-2}$ to about $200 \mathrm{k} \Omega \mathrm{cm}^{-2}$ ). A subsequent change (to about $300 \mathrm{k} \Omega \mathrm{cm}^{-2}$ ) is observed when BSA is added to block reminiscent and similar sites of anti-TLR interaction (green). Thus, both antibody and blocking agents caused a change on the charge transfer restriction of the interface indicating that the anti-TLR2 immobilization and blocking processes were successfully achieved. Subsequently, the BSA blocked receptive interfaces containing anti-TLR2 were incubated with untransfected cells and no change (from $300 \mathrm{k} \Omega \mathrm{cm}^{-2}$ ) on the $R_{c t}$ was observed (yellow). Obviously, this is thus a quantitative indication that TLR2 is not present in the untransfected cells (the differences in the signal is less than $1 \%$ lower than the experimental errors observed from the measurements into three different electrodes). Now, by tak- ing the TLR2 blocked receptive interface as the blank signal, it is possible to use the $R_{c t}$ value of this interface as referential signal to quantify the interaction of each cell with these interface. The $\mathrm{R}_{\mathrm{ct}}$ shift percentage $\left(\Delta R_{c t} \%\right)$ was thus calculated considering $\Delta \mathrm{R}_{\mathrm{ct}} \%=\left(\left(R_{c t}\right.\right.$ sample $-R_{c t}$ blank $) / R_{c t}$ blank $) \times 100$. The absolute variation (by observing the Nyquist plots) and the percentage variation are shown in Fig. 3. This Figure thus allows quantifying the percentage of interaction of anti-TLR2 antibody with TLR2 transfected cell. Fig. 3 additionally demonstrate that untransfected cells and empty vector response are appreciably different from quantitative point of view statistically demonstrated by the error bars. In summary, the optical confocal label based technique is besides label based not quantitative and is time consuming and the introduced use of label free and quantitative electrochemical EIS is demonstrated herein to be an important alternative to study general biological events involving cell receptors.

\section{Conclusions}

It has been demonstrated that label free EIS methods possess an intrinsic ability to sensitively monitor a biological event of interest including those involved with Toll-like immune receptors evaluated directly in transfected human cells. It has been either shown that EIS has advantages (for instance as lower time processing of the data and consequently at the time the assay can lead to the conclusions regarding the biological event) when compared with traditional label based-imaging analytical tools sustained on confocal microscopy. The use of electrochemical EIS demonstrates that the transducer parameter (herein based on charge transfer resistance) respond quantitatively and sensitively to the receptor target interaction directly present on the biological cellular event. The electrochemical EIS has either the advantages of being simple and allows future development that can be at the same time multi parameter in nature (multiplexing capability). As essentially spectroscopic it can also be frequency optimized (to be yet faster in data acquisition and processing). Exemplified here with a biological important target, it is clear that label free electrochemical EIS approaches have their own advantages in terms of sensitivity and 
ultimately sought to provide the biologists and either medical community with an alternative analytical toolbox that allows studying important cellular biological events (such as the quantitative presence of receptors), for instance, directly on human cells.

\section{Acknowledgments}

This study was supported by the Sao Paulo State Research Agency (FAPESP). Additional financial help was provided by National Counsel of Technological and Scientific Development (CNPq) and Coordination for the Improvement of Higher Levelor Education-Personnel (CAPES). We are grateful to Mrs. Elizabete Rosa Milani for technical assistance. The confocal microscopy was performed in the Laboratory of Confocal Microscopy of the Medical School of Ribeirao Preto, USP.

\section{References}

[1] Charles A. Janeway, P.T. Mark Walport, J. Mark, Shlomchik Innate Immnity In Immunobiology: the Immune System in Health and Disease, in: E. Lawrence (Ed.), Garland Science Publishing, New York, NY, 2005, pp. 37-102.

[2] R. Medzhitov, C. Janeway Jr., Innate immune recognition: mechanisms and pathways, Immunol. Rev. 173 (1) (2000) 89-97.

[3] C.A. Janeway Pillars, Approaching the Asymptote? Evolution and Revolution in Immunology, Cold Spring Harb Symp Quant Biol. 1989, 54, 1-13, The Journal of Immunology, (2013),191 (9), 4475-4487.

[4] N.J. Gay, M. Gangloff, Structure and function of toll receptors and their ligands, Annu. Rev. Biochem. 76 (1) (2007) 141-165.

[5] N.J. Gay, M.F. Symmons, M. Gangloff, C.E. Bryant, Assembly and localization of Toll-like receptor signalling complexes, Nat. Rev. Immunol. 14 (8) (2014) 546-558.

[6] W.D. Swaim, Overview of confocal microscopy, Methods Mol. Biol. 588 (2010) 187-201.

[7] L.-I. Larsson, Immunocytochemistry: Theory and Practice, CRC Press, Boca Raton, FL, 2000

[8] M. Ono, T. Murakami, A. Kudo, M. Isshiki, H. Sawada, A. Segawa, Quantitative comparison of anti-fading mounting media for confocal laser scanning microscopy, J. Histochem. Cytochem. 49 (3) (2001) 305-311.

[9] F.C.B. Fernandes, M.S. Goes, J.J. Davis, P.R. Bueno, Label free redox capacitive biosensing, Biosens. Bioelectron. 50 (2013) 437-440.
[10] F.C.B. Fernandes, A. Santos, D.C. Martins, M.S. Goes, P.R. Bueno, Comparing label free electrochemical impedimetric and capacitive biosensing architectures, Biosens. Bioelectron. 57 (2014) 96-102.

[11] Z. She, K. Topping, M.H. Shamsi, N.W.C. Chan, H.-B. Kraatz, Investigation of the utility of complementary electrochemical detection techniques to examine the in vitro affinity of bacterial flagellins for a toll-like receptor 5 biosensor, Anal. Chem. 87 (8) (2015) 4218-4224.

[12] H. Zhang, Z. She, H. Su, K. Kermanb, H.-B. Kraatz, Effects of bipyramidal gold nanoparticles and gold nanorods on the detection of immunoglobulins, Analyst (2016).

[13] M.N. Alam, M.H. Shamsi, H.B. Kraatz, Scanning positional variations in single-nucleotide polymorphism of DNA: an electrochemical study, Analyst 137.18 (2012) 4220-4225.

[14] Y. Li, R. Afrasiabi, F. Fathi, N. Wang, C. Xiang, R. Love, Z. She, H.-B. Kraatz, et al., Impedance based detection of pathogenic E. coli 0157: H7 using a ferrocene-antimicrobial peptide modified biosensor, Biosens. Bioelectron. 58 (2014) 193-199.

[15] A. Santos, F.C. Carvalho, M.-C. Roque-Barreira, P.R. Bueno, Impedance-derived electrochemical capacitance spectroscopy for the evaluation of lectin-glycoprotein binding affinity, Biosens. Bioelectron. 62 (2014) 102-105.

[16] V.S. Mariano, A.L. Zorzetto-Fernandes, T.A. da Silva, L.P. Ruas, L.L. Nohara, I.C. de Almeida, M.C. Roque-Barreira, Recognition of TLR2 N-Glycans: critical role in ArtinM immunomodulatory activity, PLoS One 9 (6) (2014) e98512.

[17] J. Wang, G.D. Liu, M.R. Jan, Ultrasensitive electrical biosensing of proteins and DNA: Carbon-nanotube derived amplification of the recognition and transduction events, J. Am. Chem. Soc. 126 (10) (2004) 3010-3011.

[18] F.C.B. Fernandes, A. Patil, P.R. Bueno, J. Davis, Optimized diagnostic assays based on redox tagged bio-receptive interfaces, Anal. Chem. 87 (2015) 12137-12144.

[19] A. Patil, F.C.B. Fernandes, P.R. Bueno, J.J. Davis, Immittance electroanalysis in diagnostics, Anal. Chem. 87 (2015) 944-950.

[20] F.L. Graham, J. Smiley, W.C. Russell, R. Nairn, Characteristics of a human cell line transformed by DNA from human adenovirus type 5, J. Gen. Virol. 36 (1) (1977) 59-72.

[21] A.R. Aricescu, R.J. Owens, Expression of recombinant glycoproteins in mammalian cells: towards an integrative approach to structural biology, Curr. Opin. Struct. Biol. 23 (3) (2013) 345-356.

[22] P. Thomas, T.G. Smart, HEK293 cell line: a vehicle for the expression of recombinant proteins, J. Pharmacol. Toxicol. Methods 51 (3) (2005) 187-200.

[23] A.L. Mattheyses, S.M. Simon, J.Z. Rappoport, Imaging with total internal reflection fluorescence microscopy for the cell biologist, J. Cell Sci. 123 (21) (2010) 3621-3628. 